\title{
ON TRIVIAL INTERSECTION OF CYCLIC SYLOW SUBGROUPS
}

\author{
HARVEY I. BLAU
}

\begin{abstract}
The classification of finite simple groups is used to prove that a cyclic Sylow subgroup of a finite simple group must be a trivial intersection set. Applications to character theory, and a necessary and sufficient condition for a cyclic Sylow subgroup of an arbitrary finite group to be a trivial intersection set, are obtained as corollaries.
\end{abstract}

1. Introduction. The main object of this paper is to prove, with the aid of the classification of all finite simple groups, the following ${ }^{1}$

THEOREM 1. If $P$ is a cyclic Sylow $p$-subgroup of a finite simple group $G$, then $P$ is a trivial intersection (T.I.) set in $G$.

The theorem is nontrivial as it applies to a cyclic Sylow subgroup which is not of prime order. Such subgroups occur frequently in finite simple groups of Lie type.

Before we prove Theorem 1, we will state some corollaries and give some examples. Throughout the paper, $p$ will be a fixed rational prime. If $P$ is a finite cyclic $p$-group, $\tilde{P}$ denotes the unique subgroup of $P$ of order $p$. By "simple", we mean, of course, "non-Abelian simple".

COROLlaRY 1. Let $G$ be a finite group with a cyclic Sylow $p$-subgroup $P$, and let $U=O_{p^{\prime}}(G)$. Then $P$ is a T.I. set in $G$ if and only if $C_{U}(P)=C_{U}(\tilde{P})$.

COROLlARY 2. Let $G$ be a finite simple group with a cyclic Sylow p-subgroup $P$. If $\chi$ is an irreducible character of $G$, then either $|P| \mid \chi(1)$ or $(p, \chi(1))=1$.

COROLlaRY 3. Let $G$ be a finite simple group with a cyclic Sylow p-subgroup $P$. If $\chi \neq 1_{G}$ is an irreducible character of $G$, then either $G \approx \operatorname{PSL}_{2}(p)$ or $\chi(1) \geq$ $|P|-2$.

Here are some instances of a group $G$ with a cyclic Sylow subgroup $P$ which is not a T.I. set. The solvable group $G$ of $[7$, VII.11.1] is one such example.

EXAMPLE 1 (DUE TO W. FEIT). Let $H$ be any finite group with a cyclic Sylow $p$-subgroup $P$ such that $|P|>p$. The exceptional characters $\chi$ in the principal $p$-block of $H$ are in bijection with a set of representatives $\{\lambda\}$ of $N_{G}(P) / C_{G}(P)$ orbits of $\operatorname{Irr}(P)-\{1\}$, such that $\chi=\chi_{\lambda} \leftrightarrow \lambda$ if and only if

$$
\chi_{\lambda_{P}}=m \rho_{P}+\varepsilon \sum_{g \in N_{G}(P) / C_{G}(P)} \lambda^{g}
$$

Received by the editors September 30, 1984.

1980 Mathematics Subject Classification. Primary 20D20, 20D05; Secondary 20C15, $20 \mathrm{G} 40$.

${ }^{1}$ I learned, just prior to submitting this paper, that Theorem 1 has also been recently obtained, independently, by G. Michler (not yet published), as part of his broader results on the possible defect groups that occur in finite simple groups. 
where integer $m \geq 0$ and $\operatorname{sign} \varepsilon= \pm 1$ are constant for all exceptional characters $\chi_{\lambda}$ in the principal block, and $\rho_{P}$ is the regular representation of $P$ [7, VII.2.17]. If $m=0$, fix $\lambda \in \operatorname{Irr}(P)-\{1\}$ such that $\tilde{P} \leq \operatorname{ker} \lambda$. If $m>0$, fix $\lambda$ as a faithful linear character of $P$. Then

$$
\left(1_{P}, \chi_{\lambda_{P}}\right)_{P}=m<\left\{\begin{array}{ll}
|P: \tilde{P}| m & \text { if } m>0) \\
\left|N_{G}(P): C_{G}(P)\right| & (\text { if } m=0)
\end{array}\right\}=\left(1_{\tilde{P}}, \chi_{\lambda_{\tilde{P}}}\right)_{\tilde{P}} .
$$

Let $q$ be any prime with $q \nmid|H|$. Then there exists a splitting field $\operatorname{GF}\left(q^{r}\right)$ for $H$, and a module $V$ for $H$ over $\operatorname{GF}\left(q^{r}\right)$ which lifts to a module in characteristic zero which yields the character $\chi_{\lambda}$ [7, III.3.3]. Then $V$ is an elementary Abelian $q$-group such that $C_{V}(P)<C_{V}(\tilde{P})$. Let $G$ be the semidirect product $V H$. Then $P$ is not a T.I. set in $G$ by (the easy direction of) Corollary 1 .

EXAMPLE 2. Let $H$ be any finite group with a cyclic Sylow $p$-subgroup $P$ such that $|P|>p$. Let $H$ act (not necessarily faithfully) as a group of permutations on a set $S$ of $m$ letters, such that $P$ acts nontrivially. Let $J$ be any nontrivial $p^{\prime}$-group and let $V$ be the direct product of $m$ copies of $J$. Let $H$ act on $V$ by permutation of coordinates. Since the nontrivial orbits of $P$ on $S$ break into properly smaller orbits for $\tilde{P}$, it is easily seen that $C_{V}(P)<C_{V}(\tilde{P})$. So if $G$ is the semidirect product $V H$, then $P$ is not a T.I. set in $G$.

ACKNOWLEDGMENTS. Most of the work for this paper was done during 19831984 at Yale University, where I was on sabbatical leave from Northern Illinois University. I thank both institutions for their support. I also thank Walter Feit, for responding to some questions of mine with the conjecture that Theorem 1 was true, and for encouraging me to use the classification of finite simple groups to prove it. I am also grateful to Roger Carter for some helpful remarks.

2. Proof of Theorem 1. The following result is both well known and elementary:

PROPOSITION (2.1). Let $P$ be a cyclic Sylow p-subgroup of a finite group $G$. If $P$ is a T.I. set in $G$, then $C_{H}(P)=C_{H}(\tilde{P})$ for all subgroups $H$ of $G$. Furthermore, $P$ is a T.I. set in $G$ if and only if $C_{G}(P)=C_{G}(\tilde{P})$.

Proof. Assume that $P$ is a T.I. set in $G$. For any $H \leq G$, it is clear that $C_{H}(P) \leq C_{H}(\tilde{P})$. If $h \in C_{H}(\tilde{P})$, then $P^{h} \cap P \geq \tilde{P}$ implies that $h \in N_{G}(P)$, by assumption. Then $h_{p^{\prime}}$ (the $p^{\prime}$-part of $h$ ) normalizes $P$ and centralizes $\tilde{P}$, hence must also centralize $P$. Also, $h_{p} \in P$. So $h=h_{p} h_{p^{\prime}} \in C_{H}(P)$, and the first statement is proved.

To obtain the second statement, we may assume that $C_{G}(P)=C_{G}(\tilde{P})$. If $\tilde{P} \leq P^{g} \cap P$ for some $g \in G$ then $P^{g} P \leq C_{G}(\tilde{P})=C_{G}(P)$, which has a unique Sylow $p$-subgroup $P$. Thus $P^{g}=P$ and $P$ is a T.I. set.

PROOF OF THEOREM 1. Assume that $G$ is a finite simple group with a cyclic Sylow $p$-subgroup $P$ of order $p^{n}$. By Burnside's transfer theorem, $p>2$.

If $G$ is an alternating group, suppose that $g \in P$ has order $p^{2}$. Then $g^{p}$ is a product of at least $p$ disjoint cycles of length $p$, and hence is not conjugate in $G$ to any power of a single $p$-cycle. So $G$ has more than one conjugacy class of subgroups of order $p$. This contradicts the assumption that $P$ is cyclic, and hence implies that $|P|=p$, so that $P$ is clearly a T.I. set. 
Suppose that $G$ is a sporadic group. Then the list of normalizers of subgroups of prime order in $G[9$, pp. 40-70] shows that $G$ has no cyclic Sylow subgroups apart from those of prime order.

By the classification of the finite simple groups (see [8, Chapter 2]), we may assume that $G$ is of Lie type (nontwisted or twisted). Let $C=C_{G}(P), \tilde{C}=C_{G}(\tilde{P})$. It suffices to prove $C=\tilde{C}$, by Proposition 2.1.

Let $G$, as a (possibly twisted) Chevalley group, be defined over the field GF $(q)$, $q$ a prime power. (When $G$ is twisted, we mean here by " $q$ " what Carter $[\mathbf{3}$, p. 251] means by " $q^{2} ", " q^{3} ", " 2^{2 m+1} "$ or " $3^{2 m+1} "$.) Suppose first that $p \mid q$. Then if $G$ is nontwisted, $P$ has distinct root subgroups $X_{s}$ for each positive root $s$, with $X_{s} \approx(\mathrm{GF}(q),+)$, the additive group of $\mathrm{GF}(q)[3,5.3 .3]$. Hence, $P$ cyclic implies that $q=p$ and $G$ has rank 1. Therefore, $G=A_{1}(p)=\mathrm{PSL}_{2}(p)$ and $|P|=p$, hence $P$ is a T.I. set. If $G$ is twisted, then $P$ has distinct subgroups $X_{S}^{1}$ for each equivalence class $S$ of positive roots (as in [3, Chapter 13]). Moreover, since $p>2$, we have $G \not{ }^{2} B_{2}\left(2^{2 m+1}\right),{ }^{2} F_{4}\left(2^{2 m+1}\right)$ or ${ }^{2} F_{4}(2)^{\prime}$. Then $q>p$ and $S$ has the structure of a fundamental system of a root system of type $A_{1}, A_{1} \times A_{1}$ (both roots of the same length), $A_{1} \times A_{1} \times A_{1}, A_{2}$ or $G_{2}[3,13.6 .3]$. If some $S$ has type $A_{1} \times A_{1}$ or $A_{1} \times A_{1} \times A_{1}$, then $[3,13.6 .3,13.6 .4]$ implies that $X_{S}^{1} \approx(\mathrm{GF}(q),+)$, an elementary Abelian group of order $q>p$, which contradicts our hypothesis on $P$. If some $S$ has type $G_{2}$, then by $[3,13.6 .4]$, the elements $x_{S}(0, u, v)$ of $X_{S}^{1}$ form an elementary Abelian group of order $q^{2}$, again a contradiction. If some $S$ has type $A_{2}$, then $X_{S}^{1}$ has order $q^{3 / 2}$ and exponent $p$, but cyclic $P$ has no such subgroup. Thus every class $S$ of positive roots must be of type $A_{1}$, which contradicts the fact that the underlying symmetry of a Dynkin diagram used to define the twisted group $G$ indeed has nontrivial orbits.

So we may assume that $G$ is defined over $\operatorname{GF}(q)$ with $p \nmid q$. Let $\hat{G}$ be the universal Chevalley (or twisted Chevalley) group defined over $\operatorname{GF}(q)$ such that $\hat{G} / Z(\hat{G}) \approx G$. (If $G \approx{ }^{2} F_{4}(2)^{\prime}$, we may either handle this case separately or replace $G$ by ${ }^{2} F_{4}(2)$, in which $G$ has index 2 , as it suffices to show $P$ is a T.I. set in ${ }^{2} F_{4}(2)$.) Then there exists a simply connected, simple linear algebraic group $G^{*}$, defined over the algebraic closure of $\mathrm{GF}(q)$, and an endomorphism $\sigma$ of $G^{*}$ onto $G^{*}$, such that $\hat{G}$ equals $G_{\sigma}^{*}$, the fixed-point set of $\sigma[10,11.6,12.8]$.

Let $x$ generate $P$, and let $y=x^{p^{n-1}}$, so that $\tilde{P}=\langle y\rangle$. Let $Z=Z(\hat{G})=$ $O_{p}(Z) \times O_{p^{\prime}}(Z)$. Let $\pi$ be the natural projection of $\hat{G}$ onto $G$. Let $\hat{x}$ be a $p$-element in $\hat{G}$ such that $\pi(\hat{x})=x$. Let $\hat{y}=\hat{x}^{p^{n-1}}$, so that $\pi(\hat{y})=y$.

Let $T=\pi^{-1}(\tilde{C})$. Then $T=\left\{g \in \hat{G} \mid g^{-1} \hat{y} g=\hat{y} z\right.$, some $\left.z \in Z\right\}$ and $C_{\hat{G}}(\hat{y}) \triangle T$. Now, $\hat{y}$ a $p$-element and $g^{-1} \hat{y} g=\hat{y} z$ imply that $z \in O_{p}(Z)$. Since $g^{-m} \hat{y} g^{m}=\hat{y} z^{m}$ for all integers $m$, it follows that $g^{p^{b}} \in C_{\hat{G}}(\hat{y})$ for some $b$. Thus, $T / C_{\hat{G}}(\hat{y})$ is a $p$ group. But $C_{\hat{G}}(\hat{y}) \geq\left\langle\hat{x}, O_{p}(Z)\right\rangle$, which is a Sylow $p$-subgroup of $\hat{G}$. It follows that $\pi^{-1}(\tilde{C})=C_{\hat{G}}(\hat{y})$. Thus $\tilde{C}=C_{\hat{G}}(\hat{y}) / Z$, and by a similar argument, $C=C_{\hat{G}}(\hat{x}) / Z$. So it suffices to prove that $C_{\hat{G}}(\hat{x})=C_{\hat{G}}(\hat{y})$.

A $p^{\prime}$-automorphism of $P$ must act faithfully on $\tilde{P}$. Hence, $N_{\tilde{C}}(P)=C_{\tilde{C}}(P)$. So Burnside's transfer theorem implies that $\tilde{C}$ has a normal $p$-complement, say $H$. Let $J=\pi^{-1}(H)$, so that $J / Z \approx H$. Thus $J / O_{p}(Z)$ is a $p^{\prime}$-group, and so $J=O_{p}(Z) \times V$ 
for some $p^{\prime}$-subgroup $V$, by the Schur-Zassenhaus Theorem. Thus,

$$
C_{\hat{G}}(\hat{y}) \text { has a normal } p \text {-complement } V \text {. }
$$

Since $C_{\hat{G}}(\hat{y}) / V \approx\left\langle\hat{x}, O_{p}(Z)\right\rangle$, which is Abelian, it follows that

$$
V \geq C_{\hat{G}}(\hat{y})^{\prime} .
$$

Now $p \nmid q$ implies that $\hat{y}$ is a semisimple element of $\hat{G}$ (and of $G^{*}$ ), so by $[\mathbf{1 0}$, 8.5], $C_{G^{*}}(\hat{y})$ is connected. Furthermore, $C_{G^{*}}(\hat{y})$ is a $\sigma$-stable reductive subgroup of maximal rank in $G^{*}$, and $C_{\hat{G}}(\hat{y})=C_{G^{*}}(\hat{y})_{\sigma}[\mathbf{4}, \S 1]$. Also, $C_{G^{*}}(\hat{y})=M S$, where $M$ is semisimple, $S$ is a torus central in $C_{G^{*}}(\hat{y})$, and both $M$ and $S$ are $\sigma$-stable [4, $\S 3]$. Moreover, by [5, §1],

$$
\left|C_{\hat{G}}(\hat{y})\right|=\left|M_{\sigma}\right|\left|S_{\sigma}\right| .
$$

Now by $[10,11.6,11.19,12.6], M_{\sigma}$ has the following structure: $M_{\sigma}$ contains the central product of subgroups $M_{i}, 1 \leq i \leq m$, where $\left|M_{\sigma}\right|=\prod_{i}\left|M_{i}\right|$ and each $M_{i}$ has a normal series $Z_{i} \leq R_{i} \leq M_{i}$ such that $Z_{i} \leq Z\left(M_{i}\right), M_{i} / R_{i}$ is Abelian, and $R_{i} / Z_{i}:=H_{i}$ is either a simple group of Lie type (possibly twisted) or is one of the eight finite adjoint groups which happen to be not simple (namely, $A_{1}(2) \approx$ $\mathrm{PSL}_{2}(2), A_{1}(3) \approx \mathrm{PSL}_{2}(3), B_{2}(2), G_{2}(2),{ }^{2} A_{2}\left(2^{2}\right) \approx \mathrm{PSU}_{3}(4),{ }^{2} B_{2}(2),{ }^{2} G_{2}(3)$, $\left.{ }^{2} F_{4}(2)[3,11.1 .2,14.4 .1]\right)$. The characteristic of $H_{i}$ must divide $q$. Furthermore, if $U_{i}$ is the universal Chevalley group which covers $H_{i}$, then $\left|M_{i}\right||| U_{i} \mid$. It follows from [8, Tables 2.4 and 4.1] that

$$
\text { If } r \text { is any prime divisor of }\left|M_{i}\right| \text {, then } r|| H_{i} \mid \text {. }
$$

Since $H_{i}^{\prime}$ is a section of $C_{\hat{G}}(\hat{y})^{\prime}$, it follows from (2.2) and (2.3) that $H_{i}^{\prime}$ is a $p^{\prime}$ group. So if $H_{i}$ is simple or if $H_{i} / H_{i}^{\prime}$ is a 2-group, then (2.5) implies that $M_{i}$ is a $p^{\prime}$-group.

Suppose that $p|| H_{i} \mid$ for some $i$, say $i=1$. Then $H_{1}$ is one of the eight groups above, with $p|| H_{1}: H_{1}^{\prime} \mid$. It follows that $H_{1} \approx A_{1}(3),{ }^{2} A_{2}\left(2^{2}\right)$ or ${ }^{2} G_{2}(3)$ and $p=3$ [3, pp. 176, 268]. But $\mathrm{SL}_{3}(4)$ has no element of order 9 , hence a Sylow 3-subgroup of $\mathrm{PSU}_{3}(4)$ is elementary Abelian of order 9 . So $H_{1} \not \varpi^{2} A_{2}\left(2^{2}\right)$, as $P$ is cyclic. If $H_{1} \approx{ }^{2} G_{2}(3)$, then 3||$^{2} G_{2}(3)^{\prime} \mid\left[3\right.$, p. 268], which contradicts the fact that $H_{1}^{\prime}$ is a $p^{\prime}$-group. Thus, $H_{1} \approx A_{1}(3)$. But this forces 3 (the characteristic) to divide $q$, which contradicts $p \nmid q$.

It follows that $H_{i}$ is a $p^{\prime}$-group for $1 \leq i \leq m$. Then $M_{\sigma}$ is a $p^{\prime}$-group by (2.5). So (2.4) implies that $\left|C_{\hat{G}}(\hat{y})\right|_{p}|| S_{\sigma} \mid$. Then $S_{\sigma}$, central in $C_{\hat{G}}(\hat{y})$, must contain all p-elements of $C_{\hat{G}}(\hat{y})$. So $\hat{x}$ is central in $C_{\hat{G}}(\hat{y})$, and hence $C_{\hat{G}}(\hat{x})=C_{\hat{G}}(\hat{y})$.

\section{Proofs of the corollaries.}

Proof of Corollary 1. If $P$ is a T.I. set then $C_{U}(P)=C_{U}(\tilde{P})$ by Proposition 2.1. Assume conversely that $C_{U}(P)=C_{U}(\tilde{P})$. If $S, x$ denotes a subset, resp. element, of $G$, then let $\bar{S}$, resp. $\bar{x}$, denote its image in $G / U$.

Now either $\bar{G}$ is a Frobenius group with kernel $\bar{P}$, or every proper normal subgroup of $\bar{G}$ contains $\bar{P}$ (see, for example, [1, Lemma 5.1]). In the first case, $\bar{P}$ is trivially a T.I. set in $\bar{G}$. In the second case, let $J$ be a minimal normal subgroup of $\bar{G}$. Since $J$, a direct product of isomorphic simple groups, must have a cyclic Sylow $p$-subgroup, it follows that $J$ is a simple group containing $\bar{P}$. Then $\bar{P}$ is a T.I. set 
in $J$, by Theorem 1 . So in any event, $\bar{P}$ is a T.I. set in $\bar{G}$. Then $C_{\bar{G}}(\bar{P})=C_{\bar{G}}(\overline{\tilde{P}})$ by Proposition 2.1 .

Let $g \in C_{G}(\tilde{P})$. Then $\bar{g} \in C_{\bar{G}}(\bar{P})$. So if $P=\langle x\rangle$, we have $g^{-1} x g=x u$, some $u \in U$. Now $x u$ is a $p$-element of $U P$, hence equals $v^{-1} x^{i} v$ for some $v \in U$ and integer $i$. But $x u \in x U$ and $v^{-1} x^{i} v \in x^{i} U$ imply that $x^{i}=x$. So $g^{-1} x g=v^{-1} x v$, some $v \in U$. Thus $v g^{-1} \in C_{G}(P) \leq C_{G}(\tilde{P})$, whence $v \in C_{U}(\tilde{P})=C_{U}(P)$. It follows that $g \in C_{G}(P)$. Hence, $P$ is a T.I. set in $G$ by Proposition 2.1.

PROOF OF COROLlaRY 2. A defect group $D$ of the $p$-block containing $\chi$ may be written as $P^{g} \cap P$ for some $g \in G[7$, III.8.14]. Then $D=\langle 1\rangle$ or $P$ by Theorem 1. The result follows by $[\mathbf{7}$, IV.4.5 and VII.2.16].

PROOF OF COROLlaRY 3. If $|P|=p$, the result is immediate from $[6$, Theorem 1] (see also the remarks of $[6$, p. 378] regarding the case $p<7$ ). If $|P|>p$, the result follows from Corollary 2 and [2, Theorem 1].

\section{REFERENCES}

1. H. I. Blau, On Brauer stars, J. Algebra 90 (1984), 169-188.

2. __ Character degrees of groups with a cyclic Sylow subgroup, Comm. Algebra 13 (1985), 419-463.

3. R. W. Carter, Simple groups of Lie type, Wiley-Interscience, New York, 1972.

4. _ Centralizers of semisimple elements in finite groups of Lie type, Proc. London Math. Soc. 37 (1978), 491-507.

5. __ Centralizers of semisimple elements in the finite classical groups, Proc. London Math. Soc. 42 (1981), 1-42.

6. W. Feit, On finite linear groups, J. Algebra 5 (1967), 378-400.

7. _ The representation theory of finite groups, North-Holland, Amsterdam, 1982.

8. D. Gorenstein, Finite simple groups, Plenum Press, New York, 1982.

9. D. Gorenstein and R. Lyons, The local structure of finite groups of characteristic 2 type, Mem. Amer. Math. Soc. No. 276 (1983).

10. R. Steinberg, Endomorphisms of linear algebraic groups, Mem. Amer. Math. Soc. No. 80 (1968).

Department of MATHEMATICAL SCIENCES, NORTHERN Illinois UNiversity, DEKALB, ILLINOIS 60115 